Article

\title{
Oscillatory Properties of Third-Order Neutral Delay Differential Equations with Noncanonical Operators
}

\author{
George E. Chatzarakis ${ }^{1}$, Jozef Džurina ${ }^{2}$ and Irena Jadlovská ${ }^{2, *}$
}

1 Department of Electrical and Electronic Engineering Educators, School of Pedagogical and Technological Education (ASPETE), 14121 Athens, Greece; geaxatz@otenet.gr

2 Department of Mathematics and Theoretical Informatics, Faculty of Electrical Engineering and Informatics, Technical University of Kosice, Letna 9, 04200 Kosice, Slovakia; jozef.dzurina@tuke.sk

* Correspondence: irena.jadlovska@tuke.sk

Received: 24 September 2019; Accepted: 19 November 2019; Published: 3 December 2019

check for updates

\begin{abstract}
In the paper, we study the oscillatory and asymptotic properties of solutions to a class of third-order linear neutral delay differential equations with noncanonical operators. Via the application of comparison principles with associated first and second-order delay differential inequalities, we offer new criteria for the oscillation of all solutions to a given differential equation. Our technique essentially simplifies the process of investigation and reduces the number of conditions required in previously known results. The strength of the newly obtained results is illustrated on the Euler type equations.
\end{abstract}

Keywords: linear differential equation; delay; third-order; noncanonical operators; oscillation

MSC: 34C10; 34K11

\section{Introduction}

This paper deals with the oscillatory behavior of solutions to a third-order linear neutral delay differential equation of the form

$$
\left(r_{2}(t)\left(r_{1}(t) y^{\prime}(t)\right)^{\prime}\right)^{\prime}+q(t) x(\sigma(t))=0, \quad t \geq t_{0}>0,
$$

where $y(t)=x(t)+p(t) x(\tau(t))$. We make the following assumptions:

Hypothesis 1 (H1). $r_{i} \in \mathcal{C}^{(3-i)}\left(\left[t_{0}, \infty\right),(0, \infty)\right), i=1,2$;

Hypothesis $2(\mathrm{H} 2) . p, q \in \mathcal{C}\left(\left[t_{0}, \infty\right),[0, \infty)\right)$, $q$ does not vanish eventually, and $p(t) \geq p_{0}>1$;

Hypothesis $3(\mathrm{H} 3) . \tau \in \mathcal{C}^{1}\left(\left[t_{0}, \infty\right), \mathbb{R}\right)$ is strictly increasing, $\tau(t) \leq t$, and $\lim _{t \rightarrow \infty} \tau(t)=\infty ; \sigma \in$ $\mathcal{C}\left(\left[t_{0}, \infty\right), \mathbb{R}\right), \sigma(t) \leq t$, and $\lim _{t \rightarrow \infty} \sigma(t)=\infty$;

Hypothesis $4(\mathrm{H} 4) . h(t):=\tau^{-1}(\sigma(t)) \leq t$.

For the sake of simplicity, we define the operators

$$
L_{0} y=y, \quad L_{1} y=r_{1} y^{\prime}, \quad L_{2} y=r_{2}\left(r_{1} y^{\prime}\right)^{\prime}, \quad L_{3} y=\left(r_{2}\left(r_{1} y^{\prime}\right)^{\prime}\right)^{\prime},
$$


and assume without further mention that $L_{3} y$ is of noncanonical type, that is,

$$
\int_{t_{0}}^{\infty} \frac{\mathrm{d} s}{r_{1}(s)}<\infty \text { and } \int_{t_{0}}^{\infty} \frac{\mathrm{d} s}{r_{2}(s)}<\infty
$$

Under a solution of Equation (1), we mean a nontrivial function $x \in \mathcal{C}^{1}\left(\left[T_{y}, \infty\right), \mathbb{R}\right)$ with $T_{y} \geq t_{0}$, which has the property $L_{i} y \in \mathcal{C}^{1}\left(\left[T_{y}, \infty\right), \mathbb{R}\right), i=0,1,2$, and satisfies $(1)$ on $\left[T_{y}, \infty\right)$. We restrict our attention to only those solutions of (1) which exist on some half-line $\left[T_{y}, \infty\right)$ and satisfy the condition

$$
\sup \{|y(t)|: T \leq t<\infty\}>0 \quad \text { for any } \quad T \geq T_{y} .
$$

We tacitly assume that (1) admits such a solution. A solution $y$ of (1) is called oscillatory if it has arbitrarily large zeros and nonoscillatory otherwise. The equation itself is termed oscillatory if all its solutions oscillate. Following the seminal work of Kiguradze and Kondrat'ev (see, e.g., [1]), we say that (1) has property $A$ if any solution $x$ of (1) is either oscillatory or satisfies $\lim _{t \rightarrow \infty} x(t)=0$.

Being aware of numerous indications of the practical importance of third-order differential equations as well as a number of mathematical problems involved [2], the area of the qualitative theory for such equations has attracted a large portion of research interest in the last three decades. The asymptotic properties of equations of type (1) with $p \equiv 0$ were extensively investigated in the literature, see, e.g., [3-14] and the references cited therein. Most of the papers have been devoted to the examination of so-called canonical equations, where conditions opposite to (2) hold, namely,

$$
\int_{t_{0}}^{\infty} \frac{\mathrm{d} s}{r_{1}(s)}=\int_{t_{0}}^{\infty} \frac{\mathrm{d} s}{r_{2}(s)}=\infty
$$

The advantage and usefulness of a noncanonical representation of linear disconjugate operators in the study of the oscillatory and asymptotic behavior of (1) was recently shown in [15]. In 2018, Džurina and Jadlovská [16] considered a particular case of Equation (1) in nonocanical form with $p \equiv 0$ and established various oscillation criteria for Equation (1). Their method simplifies the process and reduces the number of conditions required in previously known results. A further improvement of these results was presented in [17].

Depending on various ranges of $p$, a variety of results for property A of (1), its generalizations or particular cases, exist in the literature, see, e.g., [13,18-27] and the references cited therein. Among them, only the work in [13] considered (1) in noncanonical form with $0 \leq p<1$.

The question concerning the study of sufficient conditions which guarantee that all solutions of (1) in the presence of a neutral term oscillate was open until recently. First such results were established in [28] for (1) in canonical form, under the crucial requirement of $0 \leq p(t) \leq p_{0}<\infty$ and the commutativity of the delayed arguments $\tau \circ \sigma=\sigma \circ \tau$. To the best of the authors' knowledge, there is nothing known regarding property $\mathrm{A}$ or the oscillation of all solutions of (1) under the assumptions $\left(\mathrm{H}_{1}\right)-\left(\mathrm{H}_{4}\right)$. Motivated by this observation, we attempt to fill this gap by extending the ideas exploited in [16], based on comparisons with associated first and second-order delay differential inequalities, for more general neutral equations in noncanonical form.

Hopefully, our contribution should be of interest to the reader as, contrary to the majority of results in the literature, we attain the oscillation of all solutions of (1), and find the conditions of all theorems very simple and easy to verify. 


\section{Main Results}

For the reader's convenience, we list the functions to be used in the paper. That is, for $t \geq t_{1} \geq t_{0}$, we put

$$
\begin{array}{rrr}
R_{1}(t):=\int_{t_{1}}^{t} \frac{\mathrm{d} s}{r_{1}(s)}, & R_{2}(t)=\int_{t_{1}}^{t} \frac{\mathrm{d} s}{r_{2}(s)}, & R(t):=\int_{t_{1}}^{t} \frac{R_{2}(s)}{r_{1}(s)} \mathrm{d} s, \\
\pi_{1}(t):=\int_{t}^{\infty} \frac{\mathrm{d} s}{r_{1}(s)}, & \pi_{2}(t):=\int_{t}^{\infty} \frac{\mathrm{d} s}{r_{2}(s)}, & \pi(t):=\int_{t}^{\infty} \frac{\pi_{1}(s)}{r_{2}(s)} \mathrm{d} s, \\
& R\left(t, t_{1}\right):=\int_{t_{1}}^{t} \frac{1}{r_{1}(s)} \int_{s}^{t} \frac{\mathrm{d} u}{r_{2}(u)} \mathrm{d} s, \quad \tilde{R}\left(t, t_{1}\right):=\int_{t_{1}}^{t} \frac{1}{r_{1}(s)} \int_{s}^{t} \frac{\mathrm{d} u}{r_{2}(u) u^{\gamma}} \mathrm{d} s,
\end{array}
$$

where $\gamma$ is a constant satisfying

$$
0 \leq \frac{p_{0} \gamma}{p_{0}-1} \leq \frac{t q(t) R(t, h(t))}{p(h(t))}
$$

Here, we remark that the function $h$ from $\left(\mathrm{H}_{4}\right)$ is nondecreasing with $\lim _{t \rightarrow \infty} h(t)=\infty$, which follows from $h(t) \geq \sigma(t)$ and

$$
h^{\prime}(t)=\frac{\sigma^{\prime}(t)}{\tau^{\prime}\left(\tau^{-1}(\sigma(t))\right)} \geq 0
$$

respectively. As usual, all functional inequalities are assumed to hold eventually, that is, they are satisfied for all $t$ large enough.

We start with the following auxiliary result, which can be considered as a slight extension of [16] (Lemma 1) given for (1) with $p(t)=0$.

Lemma 1. Suppose that $\left(H_{1}\right)-\left(H_{3}\right)$ are satisfied and $x$ is an eventually positive solution of Equation (1). Then

$$
y(t)>x(t) \geq \frac{1}{p\left(\tau^{-1}(t)\right)}\left(y\left(\tau^{-1}(t)\right)-\frac{y\left(\tau^{-1}\left(\tau^{-1}(t)\right)\right)}{p\left(\tau^{-1}\left(\tau^{-1}(t)\right)\right)}\right)
$$

and the corresponding function $y$ belongs to one of the following classes

$$
\begin{aligned}
& y(t) \in \mathcal{S}_{1} \Longleftrightarrow y>0, \quad L_{1} y<0, \quad L_{2} y<0, \\
& y(t) \in \mathcal{S}_{2} \quad \Longleftrightarrow \quad y>0, \quad L_{1} y<0, \quad L_{2} y>0, \\
& y(t) \in \mathcal{S}_{3} \Longleftrightarrow y>0, \quad L_{1} y>0, \quad L_{2} y>0, \\
& y(t) \in \mathcal{S}_{4} \Longleftrightarrow y>0, \quad L_{1} y>0, \quad L_{2} y<0,
\end{aligned}
$$

eventually.

Proof. Choose $t_{1} \in\left[t_{0}, \infty\right)$ such that $x(\sigma(t))>0$ and $x(\tau(t))>0$ on $\left[t_{1}, \infty\right)$. From the definition of $y$, we see that $y(t)>x(t)>0$ and

$$
x(t)=\frac{y\left(\tau^{-1}(t)\right)-x\left(\tau^{-1}(t)\right)}{p\left(\tau^{-1}(t)\right)} \geq \frac{1}{p\left(\tau^{-1}(t)\right)}\left(y\left(\tau^{-1}(t)\right)-\frac{y\left(\tau^{-1}\left(\tau^{-1}(t)\right)\right)}{p\left(\tau^{-1}\left(\tau^{-1}(t)\right)\right)}\right)
$$

for $t \geq t_{1}$. Obviously, $L_{3} y$ is nonincreasing on $\left[t_{1}, \infty\right)$, since

$$
L_{3} y(t)=-q(t) x(\sigma(t)) \leq 0
$$

Hence, $L_{1} y$ and $L_{2} y$ are of one sign eventually, which implies that four cases $\mathcal{S}_{1}-\mathcal{S}_{4}$ are possible for $y$. 
Next, we state an auxiliary criterion for the nonexistence of positive increasing solutions of (1). As will be shown later, this condition is already included in those eliminating solutions from the class $\mathcal{S}_{1}$. In the proof, we will take advantage of the useful fact

$$
\lim _{t \rightarrow \infty} \frac{R\left(\tau^{-1}(t)\right)}{R(t)}=\lim _{t \rightarrow \infty} \frac{R_{1}\left(\tau^{-1}(t)\right)}{R_{1}(t)}=1,
$$

which follows from (2).

Lemma 2. Suppose that $\left(H_{1}\right)-\left(H_{3}\right)$ are satisfied. If

$$
\int_{t_{0}}^{\infty} \frac{\pi_{2}(s) q(s)}{p(h(s))} \mathrm{d} s=\infty,
$$

then $\mathcal{S}_{3}=\mathcal{S}_{4}=\varnothing$.

Proof. For the sake of contradiction, let (7) be satisfied but $y \in \mathcal{S}_{3} \cup \mathcal{S}_{4}$. Choose $t_{1} \in\left[t_{0}, \infty\right)$ such that $x(t)>0, x(\sigma(t))>0$, and $x(\tau(t))>0$ on $\left[t_{1}, \infty\right)$.

Assume first that $y \in \mathcal{S}_{3}$. Since $L_{2} y$ is decreasing, we have

$$
L_{1} y(t) \geq \int_{t_{1}}^{t} \frac{1}{r_{2}(s)} L_{2} y(s) \mathrm{d} s \geq L_{2} y(t) R_{2}(t) .
$$

Thus,

$$
\left(\frac{L_{1} y(t)}{R_{2}(t)}\right)^{\prime}=\frac{L_{2} y(t) R_{2}(t)-L_{1} y(t)}{r_{2}(t) R_{2}^{2}(t)} \leq 0 .
$$

Therefore, $L_{1} y / R_{2}$ is nonincreasing on $\left[t_{1}, \infty\right)$ and moreover, this fact yields

$$
y(t) \geq \int_{t_{1}}^{t} \frac{R_{2}(t)}{r_{1}(s) R_{2}(t)} L_{1} y(s) \mathrm{d} s \geq \frac{L_{1} y(t)}{R_{2}(t)} R(t)
$$

for $t \geq t_{1}$. Consequently, $y / R$ is nonincreasing on $\left[t_{1}, \infty\right)$ as well, since

$$
\left(\frac{y(t)}{R(t)}\right)^{\prime}=\frac{L_{1} y(t) R(t)-y(t) R_{2}(t)}{r_{1}(t) R^{2}(t)} \leq 0 .
$$

From $\tau^{-1}\left(\tau^{-1}(t)\right) \geq \tau^{-1}(t)$, we have

$$
y\left(\tau^{-1}\left(\tau^{-1}(t)\right)\right) \leq \frac{R\left(\tau^{-1}\left(\tau^{-1}(t)\right)\right)}{R\left(\tau^{-1}(t)\right)} y\left(\tau^{-1}(t)\right) .
$$

Using this in (5), we find that

$$
x(t) \geq \frac{y\left(\tau^{-1}(t)\right)}{p\left(\tau^{-1}(t)\right)}\left(1-\frac{R\left(\tau^{-1}\left(\tau^{-1}(t)\right)\right)}{R\left(\tau^{-1}(t)\right) p\left(\tau^{-1}\left(\tau^{-1}(t)\right)\right)}\right) \quad t \geq t_{1} .
$$

By virtue of $\left(\mathrm{H}_{2}\right)$ and (6), there is a $t_{2} \geq t_{1}$ such that for any constant $\varepsilon \in\left(0, p_{0}-1\right)$ and $t \geq t_{2}$,

$$
\frac{R\left(\tau^{-1}\left(\tau^{-1}(t)\right)\right)}{R\left(\tau^{-1}(t)\right) p\left(\tau^{-1}\left(\tau^{-1}(t)\right)\right)} \leq \frac{1+\varepsilon}{p_{0}}
$$

which implies

$$
x(t) \geq \frac{y\left(\tau^{-1}(t)\right)}{p\left(\tau^{-1}(t)\right)}\left(1-\frac{1+\varepsilon}{p_{0}}\right)>0
$$


Combining (9) with (1), we have

$$
\begin{aligned}
0 & \geq L_{3} y(t)+\left(1-\frac{1+\varepsilon}{p_{0}}\right) \frac{q(t)}{p(h(t))} y(h(t)) \\
& \geq L_{3} y(t)+k\left(1-\frac{1+\varepsilon}{p_{0}}\right) \frac{q(t)}{p(h(t))}
\end{aligned}
$$

where we used the fact that $y$ and $h$ are increasing, and set $k:=y\left(\tau^{-1}\left(\sigma\left(t_{2}\right)\right)\right)<y(h(t))$. Integrating (10) from $t_{2}$ to $t$, we obtain

$$
L_{2} y(t) \leq L_{2} y\left(t_{2}\right)-k\left(1-\frac{1+\varepsilon}{p_{0}}\right) \int_{t_{2}}^{t} \frac{q(s)}{p(h(s))} \mathrm{d} s .
$$

On the other hand, from (2) and (7), it follows that

$$
\int_{t_{0}}^{\infty} \frac{q(s)}{p(h(s))} \mathrm{d} s=\infty
$$

which in view of (11) contradicts the positivity of $L_{2} y$.

Now, assume that $y \in \mathcal{S}_{4}$ for $t \geq t_{1}$. Using the monotonicity of $L_{1} y$, we find

$$
y(t) \geq \int_{t_{1}}^{t} \frac{1}{r_{1}(s)} L_{1} y(s) \mathrm{d} s \geq L_{1} y(t) R_{1}(t) .
$$

Thus, one can see that

$$
\left(\frac{y(t)}{R_{1}(t)}\right)^{\prime}=\frac{L_{1} y(t) R_{1}(t)-y(t)}{r_{1}(t) R_{1}^{2}(t)} \leq 0,
$$

which implies that $y / R_{1}$ in nonincreasing on $\left[t_{1}, \infty\right)$. Hence,

$$
y\left(\tau^{-1}\left(\tau^{-1}(t)\right)\right) \leq \frac{R_{1}\left(\tau^{-1}\left(\tau^{-1}(t)\right)\right)}{R_{1}\left(\tau^{-1}(t)\right)} y\left(\tau^{-1}(t)\right)
$$

As in the first case, we use (6) to arrive at (10), which holds for any $\varepsilon>0$ and $t \geq t_{2}$ for $t_{2} \geq t_{1}$ sufficiently large. Integrating (10) from $t_{2}$ to $t$, we have

$$
-\left(L_{1} y(t)\right)^{\prime} \geq k\left(1-\frac{1+\varepsilon}{p_{0}}\right) \frac{1}{r_{2}(t)} \int_{t_{2}}^{t} \frac{q(s)}{p(h(s))} \mathrm{d} s .
$$

Integrating the above inequality again from $t_{2}$ to $t$, we find that

$$
L_{1} y(t) \leq L_{1} y\left(t_{2}\right)-k\left(1-\frac{1+\varepsilon}{p_{0}}\right) \int_{t_{2}}^{t} \frac{1}{r_{2}(u)} \int_{t_{2}}^{u} \frac{q(s)}{p(h(s))} \mathrm{d} s \mathrm{~d} u .
$$

Letting $t$ to $\infty$, changing the order of integration, and using (7), we obtain

$$
\begin{aligned}
0 \leq L_{1} y(\infty) & \leq L_{1} y\left(t_{2}\right)-k\left(1-\frac{1+\varepsilon}{p_{0}}\right) \int_{t_{2}}^{\infty} \frac{1}{r_{2}(u)} \int_{t_{2}}^{u} \frac{q(s)}{p(h(s))} \mathrm{d} s \mathrm{~d} u \\
& =L_{1} y\left(t_{2}\right)-k\left(1-\frac{1+\varepsilon}{p_{0}}\right) \int_{t_{2}}^{\infty} \frac{\pi_{2}(s) q(s)}{p(h(s))} \mathrm{d} s=-\infty
\end{aligned}
$$

a contradiction. The proof is complete.

Theorem 1. Suppose that $\left(H_{1}\right)-\left(H_{3}\right)$ are satisfied. If

$$
\int_{t_{0}}^{\infty} \frac{\pi(s) q(s)}{p(h(s))} \mathrm{d} s=\infty,
$$


then (1) has property $A$.

Proof. Assume that $x$ is a nonoscillatory solution of (1). Without loss of generality, we may make it eventually positive, i.e., we suppose that $x(t)>0, x(\sigma(t))>0$ and $x(\tau(t))>0$ on $\left[t_{1}, \infty\right)$, where $t_{1} \geq t_{0}$ is large enough. By conclusion of Lemma $1, y \in \mathcal{S}_{i}, i=1,2,3,4$ for $t \geq t_{1}$. First, it is easy to see that in view of (2), condition (12) implies

$$
\int_{t_{0}}^{\infty} \frac{\pi_{2}(s) q(s)}{p(h(s))} \mathrm{d} s=\int_{t_{0}}^{\infty} \frac{q(s)}{p(h(s))} \mathrm{d} s=\infty .
$$

Thus, by Lemma $2, \mathcal{S}_{3}=\mathcal{S}_{4}=\varnothing$ and so either $y \in \mathcal{S}_{1}$ or $y \in \mathcal{S}_{2}$. Using $\left(\mathrm{H}_{2}\right)$ and the fact that $y$ is decreasing in (5), we have

$$
x(t) \geq \frac{y\left(\tau^{-1}(t)\right)}{p\left(\tau^{-1}(t)\right)}\left(1-\frac{1}{p\left(\tau^{-1}\left(\tau^{-1}(t)\right)\right)}\right) \geq\left(1-\frac{1}{p_{0}}\right) \frac{y\left(\tau^{-1}(t)\right)}{p\left(\tau^{-1}(t)\right)} .
$$

On the other hand, since $y^{\prime}<0$, there is a constant $\ell>0$ such that

$$
\lim _{t \rightarrow \infty} y(t)=\ell<\infty
$$

If $\ell>0$, there exists a $t_{2} \geq t_{1}$ such that $y(t) \geq \ell$ for $t \geq t_{2}$. Hence, from (13), we see that

$$
x(t) \geq \frac{\ell\left(p_{0}-1\right)}{p_{0}} \frac{1}{p\left(\tau^{-1}(t)\right)}, \quad t \geq t_{2} .
$$

Using this in (1), we find

$$
L_{3} y(t)+\frac{\ell\left(p_{0}-1\right)}{p_{0}} \frac{q(t)}{p(h(t))} \leq 0, \quad t \geq t_{2}
$$

If we assume that $y \in \mathcal{S}_{1}$, then by integrating (14) from $t_{2}$ to $t$, we obtain

$$
-\left(L_{1} y(t)\right)^{\prime} \geq \frac{\ell\left(p_{0}-1\right)}{p_{0}} \frac{1}{r_{2}(t)} \int_{t_{2}}^{t} \frac{q(s)}{p(h(s))} \mathrm{d} s .
$$

Integrating the above inequality over $\left[t_{2}, t\right]$ again, we have

$$
-y^{\prime}(t) \geq \frac{\ell\left(p_{0}-1\right)}{p_{0}} \frac{1}{r_{1}(t)} \int_{t_{2}}^{t} \frac{1}{r_{2}(u)} \int_{t_{2}}^{u} \frac{q(s)}{p(h(s))} \mathrm{d} s \mathrm{~d} u .
$$

Integrating (15) over $\left[t_{2}, t\right]$, letting $t$ to $\infty$ and changing the order of integration in the resulting inequality, and taking (12) into account, we obtain

$$
\begin{aligned}
\ell=y(\infty) & \leq y\left(t_{2}\right)-\frac{\ell\left(p_{0}-1\right)}{p_{0}} \int_{t_{2}}^{\infty} \frac{1}{r_{1}(v)} \int_{t_{2}}^{v} \frac{1}{r_{2}(u)} \int_{t_{2}}^{u} \frac{q(s)}{p(h(s))} \mathrm{d} s \mathrm{~d} u \mathrm{~d} v \\
& =y\left(t_{2}\right)-\frac{\ell\left(p_{0}-1\right)}{p_{0}} \int_{t_{2}}^{\infty} \frac{\pi(s) q(s)}{p(h(s))} \mathrm{d} s=-\infty
\end{aligned}
$$

a contradiction. Hence, $\ell=0$ in this case.

If we take $y \in \mathcal{S}_{2}$, then by integrating (14) from $t_{2}$ to $t$ and using (12), we arrive at

$$
L_{2} y(t) \leq L_{2} y\left(t_{2}\right)-\frac{\ell\left(p_{0}-1\right)}{p_{0}} \int_{t_{2}}^{t} \frac{q(s)}{p(h(s))} \mathrm{d} s \rightarrow-\infty \quad \text { as } t \rightarrow \infty,
$$

which contradicts the positivity of $L_{2} y$ and so $\ell=0$. Since $y(t) \geq x(t)$, we find $\lim _{t \rightarrow \infty} x(t)=0$. The proof is complete. 
In the following auxiliary result, we present a criterion for nonexistence of $\mathcal{S}_{1}$ type solutions, based on comparison of the studied Equation (1) with an associated first-order delay differential inequality. The given criterion also excludes solutions from classes $\mathcal{S}_{3}$ and $\mathcal{S}_{4}$.

Lemma 3. Suppose that $\left(H_{1}\right)-\left(H_{4}\right)$ are satisfied. If

$$
\liminf _{t \rightarrow \infty} \int_{h(t)}^{t} \frac{q(s) \pi(s)}{p(h(s))} \mathrm{d} s>\frac{p_{0}}{\left(p_{0}-1\right) \mathrm{e}^{\prime}}
$$

then $\mathcal{S}_{1}=\mathcal{S}_{3}=\mathcal{S}_{4}=\varnothing$.

Proof. For the sake of contradiction, let (18) be satisfied but $y \in \mathcal{S}_{1} \cup \mathcal{S}_{3} \cup \mathcal{S}_{4}$. Choose $t_{1} \in\left[t_{0}, \infty\right)$ such that $x(t)>0, x(\sigma(t))>0$, and $x(\tau(t))>0$ on $\left[t_{1}, \infty\right)$.

Assume first that $y \in \mathcal{S}_{1}$. As in the proof of Theorem 1, we arrive at (13), which in view of (1) yields

$$
L_{3} y(t)+\frac{p_{0}-1}{p_{0}} \frac{q(t)}{p(h(t))} y(h(t)) \leq 0 .
$$

Define the function

$$
w(t):=\pi_{1}(t) L_{1} y(t)+y(t) .
$$

From

$$
y(t) \geq-\int_{t}^{\infty} \frac{1}{r_{1}(s)} L_{1} y(s) \mathrm{d} s \geq-L_{1} y(t) \pi_{1}(t)
$$

and

$$
w^{\prime}(t)=\pi_{1}(t)\left(L_{1} y(t)\right)^{\prime}=\frac{\pi_{1}(t)}{r_{2}(t)} L_{2} y(t)<0,
$$

we see that $w(t)$ is a strictly decreasing eventually positive function, say on $\left[t_{2}, \infty\right)$, for $t_{2} \geq t_{1}$. Using the definition of $w$ in (19), we have

$$
\left(\frac{r_{2}(t)}{\pi_{1}(t)} w^{\prime}(t)\right)^{\prime}+\frac{p_{0}-1}{p_{0}} \frac{q(t)}{p(h(t))} y(h(t)) \leq 0 .
$$

Hence, $w$ is a solution of the second-order delay differential inequality

$$
\left(\frac{r_{2}(t)}{\pi_{1}(t)} w^{\prime}(t)\right)^{\prime}+\frac{p_{0}-1}{p_{0}} \frac{q(t)}{p(h(t))} w(h(t)) \leq 0 .
$$

Similarly as before, we define the function $u$ by

$$
u(t):=\pi(t) \frac{r_{2}(t)}{\pi_{1}(t)} w^{\prime}(t)+w(t)
$$

From

$$
u^{\prime}(t)=\left(\frac{r_{2}(t)}{\pi_{1}(t)} w^{\prime}(t)\right)^{\prime} \pi(t)=L_{3} y(t) \pi(t) \leq 0
$$

and

$$
w(t) \geq-\int_{t}^{\infty} \frac{\pi_{1}(s)}{r_{2}(s)} \frac{r_{2}(s)}{\pi_{1}(s)} w^{\prime}(s) \mathrm{d} s \geq-\frac{r_{2}(t)}{\pi_{1}(t)} w^{\prime}(t) \pi(t),
$$

we conclude that $u$ is eventually positive and nonincreasing. Using the definition of $u$ in (22), it is easy to see that $u$ satisfies the first-order delay differential inequality

$$
u^{\prime}(t)+\frac{p_{0}-1}{p_{0}} \frac{q(t) \pi(t)}{p(h(t))} u(h(t)) \leq 0 .
$$


However, by [29] (Theorem 2.1.1), condition (18) ensures that the above inequality does not possess a positive solution, which is a contradiction.

To show that also $\mathcal{S}_{3}=\mathcal{S}_{4}=\varnothing$, it suffices to note that (12) is necessary for validity of (18) since otherwise, the left-hand side of (18) would equal zero. The conclusion then immediately follows from Theorem 1 . The proof is complete.

Using the Riccati transformation technique, we propose a result serving as an alternative of Lemma 3, which is applicable also in the case when $h(t)=t$.

Lemma 4. Suppose that $\left(H_{1}\right)-\left(H_{4}\right)$ are satisfied and (7) holds. If, for any $t_{1} \geq t_{0}$ large enough,

$$
\limsup _{t \rightarrow \infty} \int_{t_{1}}^{t}\left(\frac{\pi(s) q(s)}{p(h(s))}-\left(\frac{p_{0}}{p_{0}-1}\right) \frac{\pi_{1}(s)}{4 \pi(s) r_{2}(s)}\right) \mathrm{d} s>\frac{p_{0}}{p_{0}-1}
$$

then $\mathcal{S}_{1}=\mathcal{S}_{3}=\mathcal{S}_{4}=\varnothing$.

Proof. For the sake of contradiction, let (18) be satisfied but $y \in \mathcal{S}_{1} \cup \mathcal{S}_{3} \cup \mathcal{S}_{4}$. Choose $t_{1} \in\left[t_{0}, \infty\right)$ such that $x(t)>0, x(\sigma(t))>0$, and $x(\tau(t))>0$ on $\left[t_{1}, \infty\right)$.

Assume first that $y \in \mathcal{S}_{1}$. We proceed as in the proof of Lemma 3 to obtain (22), where $w$ is given by (20). Consider the function $\rho$ defined by

$$
\rho(t)=\frac{r_{2}(t) w^{\prime}(t)}{\pi_{1}(t) w(t)}, \quad t \geq t_{1}
$$

Clearly, $\rho<0$ on $\left[t_{1}, \infty\right)$. From (23), it is easy to see that

$$
-1 \leq \pi(t) \rho(t)<0 .
$$

Differentiating $\rho$ and using (22) together with (26), we have

$$
\begin{aligned}
\rho^{\prime}(t) & =\left(\frac{r_{2}(t)}{\pi_{1}(t)} w^{\prime}(t)\right)^{\prime} \frac{1}{w(t)}-\frac{r_{2}(t)\left(w^{\prime}(t)\right)^{2}}{\pi_{1}(t) w^{2}(t)} \\
& \leq-\frac{p_{0}-1}{p_{0}} \frac{q(t)}{p(h(t))} \frac{w(h(t))}{w(t)}-\frac{\pi_{1}(t)}{r_{2}(t)} \rho^{2}(t) \\
& \leq-\frac{p_{0}-1}{p_{0}} \frac{q(t)}{p(h(t))}-\frac{\pi_{1}(t)}{r_{2}(t)} \rho^{2}(t) .
\end{aligned}
$$

Multiplying both sides of (28) by $\pi(t)$ and integrating the resulting inequality from $t_{1}$ to $t$, we have

$$
\begin{aligned}
\pi(t) \rho(t) & \leq \pi\left(t_{1}\right) \rho\left(t_{1}\right)-\int_{t_{1}}^{t} \frac{\pi_{1}(s) \rho(s)}{r_{2}(s)} \mathrm{d} s-\frac{p_{0}-1}{p_{0}} \int_{t_{1}}^{t} \frac{\pi(s) q(s)}{p(h(s))} \mathrm{d} s-\int_{t_{1}}^{t} \frac{\pi(s) \pi_{1}(s)}{r_{2}(s)} \rho^{2}(s) \mathrm{d} s \\
& =\pi\left(t_{1}\right) \rho\left(t_{1}\right)-\frac{p_{0}-1}{p_{0}} \int_{t_{1}}^{t} \frac{\pi(s) q(s)}{p(h(s))} \mathrm{d} s-\int_{t_{1}}^{t} \frac{\pi(s) \pi_{1}(s)}{r_{2}(s)}\left[\left(\rho(s)+\frac{1}{2 \pi(s)}\right)^{2}-\frac{1}{4 \pi^{2}(s)}\right] \mathrm{d} s \\
& \leq-\left(\frac{p_{0}-1}{p_{0}}\right) \int_{t_{1}}^{t}\left(\frac{\pi(s) q(s)}{p(h(s))}-\left(\frac{p_{0}}{p_{0}-1}\right) \frac{\pi_{1}(s)}{4 \pi(s) r_{2}(s)}\right) \mathrm{d} s .
\end{aligned}
$$

However, in view of (27), this inequality contradicts (25). Hence, $\mathcal{S}_{1}=\varnothing$. By Lemma $2, \mathcal{S}_{3}=\mathcal{S}_{4}=\varnothing$ due to (7). The proof is complete.

Corollary 1. Suppose that $\left(H_{1}\right)-\left(H_{3}\right)$ are satisfied and (7) holds. If there is a constant $c_{*}$ such that

$$
\frac{\pi^{2}(t) q(t) r_{2}(t)}{p(h(t)) \pi_{1}(t)} \geq c_{*}>\frac{p_{0}}{4\left(p_{0}-1\right)}
$$


then $\mathcal{S}_{1}=\mathcal{S}_{3}=\mathcal{S}_{4}=\varnothing$.

To attain the oscillation of all solutions, it remains to eliminate the solutions of $\mathcal{S}_{2}$ type.

Lemma 5. Suppose that $\left(H_{1}\right)-\left(H_{4}\right)$ are satisfied. If

$$
\limsup _{t \rightarrow \infty} \int_{h(t)}^{t} \frac{q(s) R(h(t), h(s))}{p(h(s))} \mathrm{d} s>\frac{p_{0}}{p_{0}-1}
$$

then $\mathcal{S}_{2}=\varnothing$.

Proof. For the sake of contradiction, let (30) be satisfied but $y \in \mathcal{S}_{2}$. Choose $t_{1} \in\left[t_{0}, \infty\right)$ such that $x(t)>0, x(\sigma(t))>0$, and $x(\tau(t))>0$ on $\left[t_{1}, \infty\right)$. Using (13) in (1), we obtain

$$
L_{3} y(t)+\frac{p_{0}-1}{p_{0}} \frac{q(t)}{p(h(t))} y(h(t)) \leq 0 .
$$

Using the monotonicity of $L_{2} y$, one can easily verify that

$$
-L_{1} y(u) \geq L_{1} y(v)-L_{1} y(u)=\int_{u}^{v} \frac{L_{2} y(s)}{r_{2}(s)} \mathrm{d} s \geq L_{2} y(v) \int_{u}^{v} \frac{\mathrm{d} s}{r_{2}(s)}
$$

for $v \geq u \geq t_{1}$. Integrating the latter inequality from $u$ to $v \geq u$, we obtain

$$
y(u) \geq L_{2} y(v) \int_{u}^{v} \frac{1}{r_{1}(s)} \int_{s}^{v} \frac{1}{r_{2}(x)} \mathrm{d} x \mathrm{~d} s=L_{2} y(v) R(v, u) .
$$

Setting $u=h(s)$ and $v=h(t), t \geq s \geq t_{1}$ in (33), we find

$$
y(h(s)) \geq L_{2} y(h(t)) R(h(t), h(s)) .
$$

On the other hand, integrating (31) from $h(t)$ to $t$ and using (34), we see that

$$
\begin{aligned}
L_{2} y(h(t)) & \geq L_{2} y(h(t))-L_{2} y(t) \geq \frac{p_{0}-1}{p_{0}} \int_{h(t)}^{t} \frac{q(s)}{p(h(s))} y(h(s)) \mathrm{d} s \\
& \geq \frac{p_{0}-1}{p_{0}} L_{2} y(h(t)) \int_{h(t)}^{t} \frac{q(s) R(h(t), h(s))}{p(h(s))} \mathrm{d} s .
\end{aligned}
$$

Dividing the above inequality by $L_{2} y(h(t))$ and taking the lim sup on both sides of the resulting inequality as $t \rightarrow \infty$, we obtain a contradiction with (30). The proof is complete.

Here, a further improvement of Lemma 5 is made.

Lemma 6. Suppose that $\left(H_{1}\right)-\left(H_{4}\right)$ are satisfied and let $\gamma$ be a constant satisfying (4) eventually. If

$$
\limsup _{t \rightarrow \infty} h^{\gamma}(t) \int_{h(t)}^{t} \frac{q(s) \tilde{R}(h(t), h(s))}{p(h(s))} \mathrm{d} s>\frac{p_{0}}{p_{0}-1},
$$

then $\mathcal{S}_{2}=\varnothing$.

Proof. Setting $u=h(t)$ and $v=t$ in (33), we obtain

$$
y(h(t)) \geq L_{2} y(t) R(t, h(t))
$$


By (4), (31), and (36), we have

$$
\begin{aligned}
\left(t^{\gamma} L_{2} y(t)\right)^{\prime} & =\gamma t^{\gamma-1} L_{2} y(t)+t^{\gamma} L_{3} y(t) \leq \gamma t^{\gamma-1} L_{2} y(t)-\frac{p_{0}-1}{p_{0}} \frac{t^{\gamma} q(t)}{p(h(t))} y(h(t)) \\
& \leq \gamma t^{\gamma-1} L_{2} y(t)-\frac{p_{0}-1}{p_{0}} \frac{t^{\gamma} q(t) R(t, h(t))}{p(h(t))} L_{2} y(t) \\
& =t^{\gamma-1} L_{2} y(t)\left(\gamma-\frac{p_{0}-1}{p_{0}} \frac{t q(t) R(t, h(t))}{p(h(t))}\right) \leq 0 .
\end{aligned}
$$

That is, $t^{\gamma} L_{2} y(t)$ is eventually nonincreasing. From here, we obtain that

$$
-L_{1} y(u) \geq L_{1} y(v)-L_{1} y(u)=\int_{u}^{v} \frac{L_{2} y(s) s^{\gamma}}{s^{\gamma} r_{2}(s)} \mathrm{d} s \geq L_{2} y(v) v^{\gamma} \int_{u}^{v} \frac{\mathrm{d} s}{s^{\gamma} r_{2}(s)}
$$

for $v \geq u \geq t_{1}$. Proceeding as in the proof of Lemma 5 with (32) replaced by (37), one arrives at a contradiction with (35). The proof is complete.

As a main result of the paper, we have the following oscillatory criterion for (1), which is a simple consequence of Lemmas 3-6.

Theorem 2. Suppose that $\left(H_{1}\right)-\left(H_{4}\right)$ are satisfied. If (18) (or (25)) and (30) (or (35)) hold, then (1) is oscillatory.

Example 1. Consider the third-order neutral differential equation

$$
\left(t^{\beta}\left(t^{\alpha}\left(x(t)+p_{0} x(\tau(t))^{\prime}\right)^{\prime}\right)^{\prime}+q_{0} t^{\delta} x(\sigma(t))=0, \quad t \geq 1,\right.
$$

where $\alpha>1, \beta>1, \delta>0, q_{0}>0, p_{0}>1$, and the delay functions $\tau$ and $\sigma$ satisfy $\left(H_{3}\right)$. Using that

$$
\pi(t)=\frac{1}{(\alpha-1)(\alpha+\beta-2) t^{\alpha+\beta+2}}
$$

it is easy to verify that condition (12) reduces to

$$
\int_{1}^{\infty} \frac{q_{0}}{p_{0}(\alpha-1)(\alpha+\beta-2)} \frac{1}{t^{\alpha+\beta-2-\delta}} \mathrm{d} t=\infty
$$

Hence, if

$$
\delta \geq \alpha+\beta-3
$$

all assumptions of Theorem 1 are satisfied and (38) has property $A$.

Example 2. Consider the third-order neutral differential equation

$$
\left(t^{2}\left(t^{2}\left(x(t)+k t^{\alpha} x\left(\lambda_{1} t\right)\right)^{\prime}\right)^{\prime}\right)^{\prime}+q_{0} t^{\alpha+1} x\left(\lambda_{2} t\right)=0, \quad t \geq 1,
$$

where $k>0, \alpha \geq 0, \lambda_{1} \in(0,1], \lambda_{2} \in(0,1]$, and $h(t):=\lambda_{2} / \lambda_{1} t \leq t$. Here,

$$
\pi(t)=\frac{1}{2 t^{2}} \quad \text { and } \quad R(h(t), h(s))=\frac{1}{2}\left(\frac{\lambda_{1}}{\lambda_{2}}\right)^{2}\left(\frac{1}{t^{2}}-\frac{2}{t s}+\frac{1}{s^{2}}\right) .
$$


Since (12) is satisfied, by Theorem 1, (39) has property A. By some computations, conditions (18) and (25) reduce to

$$
\frac{q_{0}}{2}\left(\frac{\lambda_{1}}{\lambda_{2}}\right)^{\alpha} \ln \left(\frac{\lambda_{1}}{\lambda_{2}}\right)> \begin{cases}\frac{k^{2}}{(k-1) \mathrm{e}} & \text { for } \alpha=0 \text { and } k>1 \\ \frac{k}{\mathrm{e}} & \text { for } \alpha>0\end{cases}
$$

and

$$
q_{0}\left(\frac{\lambda_{1}}{\lambda_{2}}\right)^{\alpha}>\left\{\begin{array}{cl}
\frac{k^{2}}{(k-1)} & \text { for } \alpha=0 \text { and } k>1 \\
k & \text { for } \alpha>0
\end{array}\right.
$$

respectively. Finally, condition (30) takes the form

$$
\frac{q_{0}}{2}\left(\frac{\lambda_{1}}{\lambda_{2}}\right)^{2+\alpha}\left(\frac{1-\left(\frac{\lambda_{2}}{\lambda_{1}}\right)^{2}}{2}-2\left(1-\left(\frac{\lambda_{2}}{\lambda_{1}}\right)\right)+\ln \left(\frac{\lambda_{1}}{\lambda_{2}}\right)\right)>\left\{\begin{array}{cl}
\frac{k^{2}}{k-1} & \text { for } \alpha=0 \text { and } k>1 \\
k & \text { for } \alpha>0 .
\end{array}\right.
$$

By Theorem 2, we conclude that (39) is oscillatory if (40) (or (41)) and (42) hold.

Author Contributions: Investigation, I.J., J.D. and G.E.C.; Methodology, I.J., J.D. and G.E.C.; Writing â original draft, I.J., J.D. and G.E.C.; Writing review editing, I.J., J.D. and G.E.C.

Funding: This research received no external funding

Acknowledgments: The authors would like to express their sincere gratitude to the editors and two anonymous referees for the careful reading of the original manuscript and valuable suggestions that helped to improve the paper.

Conflicts of Interest: The authors declare no conflict of interest

\section{References}

1. Kiguradze, I.T.; Chanturia, T.A. Asymptotic properties of solutions of nonautonomous ordinary differential equations. In Mathematics and Its Applications (Soviet Series); Kluwer Academic Publishers Group: Dordrecht, The Netherlands, 1993; Volume 89, Translated from the 1985 Russian original.

2. Hale, J.K. Functional differential equations. In Applied Mathematical Sciences; Springer: New York, NY, USA, 1971; Volume 3.

3. Agarwal, R.; Bohner, M.; Li, T.; Zhang, C. Oscillation of third-order nonlinear delay differential equations. Taiwan J. Math. 2013, 17, 545-558. [CrossRef]

4. Agarwal, R.P.; Aktas, M.F.; Tiryaki, A. On oscillation criteria for third order nonlinear delay differential equations. Arch. Math. (Brno) 2009, 45, 1-18.

5. Baculíková, B.; Džurina, J. Oscillation of third-order functional differential equations. Electron. J. Qual. Theory Differ. Equ. 2010, 2010, 1-10. [CrossRef]

6. Baculíková, B.; Džurina, J.; Rogovchenko, Y.V. Oscillation of third order trinomial delay differential equations. Appl. Math. Comput. 2012, 218, 7023-7033. [CrossRef]

7. Bohner, M.; Grace, S.R.; Jadlovská, I. Oscillation criteria for third-order functional differential equations with damping. Electron. J. Differ. Equ. 2016, 2016, 15.

8. Candan, T.; Dahiya, R.S. Oscillation of third order functional differential equations with delay. Electron. J. Differ. Equ. Conf. 2001, 10, 79-88.

9. Chatzarakis, G.E.; Grace, S.R.; Jadlovská, I. Oscillation criteria for third-order delay differential equ. Adv. Differ. Equ. 2017, 2017, 330. [CrossRef]

10. Elabbasy, E.M.; Hassan, T.S.; Elmatary, B.M. Oscillation criteria for third order delay nonlinear differential equations. Electron. J. Qual. Theory Differ. Equ. 2012, 2012, 11. [CrossRef]

11. Grace, S.R.; Agarwal, R.P.; Pavani, R.; Thandapani, E. On the oscillation of certain third order nonlinear functional differential equations. Appl. Math. Comput. 2008, 202, 102-112. [CrossRef] 
12. Li, T.; Rogovchenko, Y.V. On asymptotic behavior of solutions to higher-order sublinear emden-fowler delay differential equations. Appl. Math. Lett. 2017, 67, 53-59. [CrossRef]

13. Li, T.; Zhang, C.; Xing, G. Oscillation of third-order neutral delay differential equations. In Abstract and Applied Analysis; Hindawi: London, UK, 2012; Volume 2012.

14. Zhang, C.; Li, T.; Sun, B.; Thandapani, E. On the oscillation of higher-order half-linear delay differential equations. Appl. Math. Lett. 2011, 24, 1618-1621. [CrossRef]

15. Baculíková, B.; Džurina, J.; Jadlovská, I. On asymptotic properties of solutions to third-order delay differential equations. Electron. J. Qual. Theory Differ. 2019, 2019, 1-11.

16. Džurina, J.; Jadlovská, I. Oscillation of third-order differential equations with noncanonical operators. Appl. Math. Comput. 2018, 336, 394-402. [CrossRef]

17. Grace, S.R.; Jadlovská, I.; Zafer, A. On oscillation of third-order noncanonical delay differential equations. Appl. Math. Comput. 2019, 362, 124530. [CrossRef]

18. Baculíková, B.; Džurina, J. Oscillation of third-order neutral differential equations. Math. Comput. Model. 2010, 52, 215-226. [CrossRef]

19. Došlá, Z.; Lixsxka, P. Comparison theorems for third-order neutral differential equ. Electron. J. Differ. Equ. 2016, 2016, 13.

20. Došlá, Z.; Lixsxka, P. Oscillation of third-order nonlinear neutral differential equations. Appl. Math. Lett. 2016, 56, 42-48. [CrossRef]

21. Dzurina, J.; Thandapani, E.; Tamilvanan, S. Oscillation of solutions to third-order half-linear neutral differential equations. Electron. J. Differ. Equ. 2012, 2012, 1-9.

22. Graef, J.R.; Tunc, E.; Grace, S.R. Oscillatory and asymptotic behavior of a third-order nonlinear neutral differential equation. Opusc. Math. 2017, 37, 839-852. [CrossRef]

23. Han, Z.; Li, T.; Sun, S.; Zhang, C. Oscillation behavior of third-order neutral emden-fowler delay dynamic equations on time scales. Adv. Differ. Equ. 2010, 2010, 586312. [CrossRef]

24. Jiang, Y.; Li, T. Asymptotic behavior of a third-order nonlinear neutral delay differential equation. J. Inequal. Appl. 2014, 2014, 512. [CrossRef]

25. Thandapani, E.; Li, T. On the oscillation of third-order quasi-linear neutral functional differential equations. Arch. Math. 2011, 47, 181-199.

26. Thandapani, E.; Tamilvanan, S.; Jambulingam, E.; Tech, V.T.M. Oscillation of third order half linear neutral delay differential equations. Int. J. Pure Appl. Math. 2012, 77, 359-368.

27. Tunc, E. Oscillatory and asymptotic behavior of third-order neutral differential equations with distributed deviating arguments. Electron. J. Differ. Equ. 2017. [CrossRef]

28. Džurina, J.; Grace, S.R.; Jadlovská, I. On nonexistence of Kneser solutions of third-order neutral delay differential equations. Appl. Math. Lett. 2019, 88, 193-200. [CrossRef]

29. Ladde, G.S.; Lakshmikantham, V.; Zhang, B.G. Oscillation theory of differential equations with deviating arguments. In Monographs and Textbooks in Pure and Applied Mathematics; Marcel Dekker, Inc.: New York, NY, USA, 1987; Volume 110.

(C) 2019 by the authors. Licensee MDPI, Basel, Switzerland. This article is an open access article distributed under the terms and conditions of the Creative Commons Attribution (CC BY) license (http://creativecommons.org/licenses/by/4.0/). 